\title{
BlueSPACE's SDN/NFV Architecture for 5G SDM/WDM-enabled Fronthaul with Edge Computing
}

\author{
Raul Muñoz, Ricard Vilalta, Josep M. Fàbrega, Laura \\ Rodríguez, Ricardo Martínez, Ramon Casellas \\ Centre Tecnològic de Telecomunicacions de Catalunya \\ (CTTC/CERCA) \\ Castelldefels (Barcelona), Spain \\ raul.munoz@cttc.es
}

\author{
Giada Landi, Marco Capitani \\ Nextworks \\ Via Livornese 1027 \\ Pisa, Italy \\ \{g.landi,m.capitani\}@nextworks.it
}

\begin{abstract}
The BlueSPACE project proposes Spatial Division Multiplexing (SDM) as the key technology to overcome the capacity crunch that the conventional optical single-mode fibers (SMFs) are facing to accommodate the increasing bandwidth demands forecasted for $5 \mathrm{G}$ mobile communications in the fronthaul network. The combination of SDM with flexi-grid DWDM enables to exploit both dimensions (spectral and spatial resources) in combination with analog and digital Radio over Fiber (RoF) techniques. Additionally, BlueSPACE also considers the deployment of edge computing for MEC applications and VNFs. This paper presents the challenges and architectural solutions proposed to enhance the ETSI NFV MANO framework in order to meet the functionalities considered in BlueSPACE.
\end{abstract}

Keywords-SDM, $5 G$ fronthaul, NFV, Transport SDN, MEC

\section{INTRODUCTION}

Mobile transport networks can be traditionally sorted out as backhaul and fronthaul solutions. The mobile backhaul is the transport connection/network providing support to the backhaul interface between the base-band units (BBUs) and the mobile core (i.e., EPC). On the other hand, the mobile fronthaul is the transport connection/network supporting the fronthaul interface between the remote radio head (RRH) and the BBU. Mobile fronthaul has very strict requirements in terms of highbandwidth and low-delay because it is required to transport the digitally sampled radio waveform from the RRH in the base stations to the central office $(\mathrm{CO})$ where the pool of BBUs is located. The most widely used standard interface for the fronthaul is the common public radio interface (CPRI) and is deployed in C/DWDM links/networks, making use of optical transceivers with digital radio over fiber $(\mathrm{dRoF})$ solutions. The main drawback of CPRI is that it does not scale, in terms of bandwidth requirements, for the massive MIMO antenna deployments foreseen in $5 \mathrm{G}$ [1].

3GPP is proposing to reduce the bandwidth and latency requirements for $5 \mathrm{G}$, while keeping most of the benefits of the C-RAN architectures, by performing a function split of the baseband processing in eight options that have been analyzed thoroughly in literature [2]. In this approach, the BBU functions are split into three logical entities; remote unite (RU), central unit (CU) and distributed unit (DU). It brings the introduction of the next generation fronthaul interface (NGFI) that is split into two network segments. The fronthaul segment between the RU and DU is known as NGFI-I, and the network

Work supported by EC H2020 BLUESPACE (762055) and the Spanish DESTELLO (TEC2015-69256-R) projects. segment between the DU and the CU is the NGFI-II (also known as midhaul). 3GPP has already chosen the split option 2 (i.e. split between PDCP and RLC) for NGFI-II, but the splitting between DU and RU is still open. This approach enables the packetization of the NGFI to provide more efficient network utilization in ultra-dense scenarios, and opens the door to virtualize BBU functions (e.g., CU).

An alternative solution under investigation to reduce the bandwidth requirements in the BlueSPACE project is to use analog radio over fibre ( $\mathrm{aRoF})$ transceivers, where the radio waveforms are directly modulated onto light for connecting BBUs and RRHs. In this scenario, BBUs are not virtualized and they are considered Physical Network Functions (PNFs). Additionally, the considered optical network architecture in BlueSPACE envisions the gradual upgrade of the fronthaul network in order to include spatial division multiplexing (SDM) for further increasing the network capacity. Therefore, the spatial diversity obtained, in combination with WDM, would enable a fronthaul infrastructure with unique 2dimensional (2D) properties. A first approach for SDM can be based on bundles of standard single-mode fibers (SSMFs), since cables deployed in the field typically have a loose-tube design containing several fibers. Nevertheless, a longer-term solution will rely on multicore fibers (MCFs), providing a compact parallel transmission medium.

Additionally, the wide adoption of Network Function Virtualization (NFV) concepts (e.g. virtualization of the mobile EPC) and Mobile Edge Computing (MEC) require computing resources integrated with the NGFI-II/backhaul network for the deployment of virtualized network functions (VNFs) and MEC applications (e.g. video analytics, data caching, location services.). In addition to the computing services offered in large data centres (DCs) in the core network, it is also required to offer computing services at the edge of the network for applications with very stringent delay requirements. The adoption of a reference architecture such as the ETSI NFV Management and Orchestration (MANO) framework [3] can provide an efficient network service management and resource orchestration from an end-to-end perspective. However, it is required to extend the current NFV MANO framework in order to meet the new functionalities considered in the proposed $5 \mathrm{G}$ SDM/WDM-enabled fronthaul. This paper presents the challenges and requirements of the extended NFV MANO framework developed in the BlueSPACE project. 


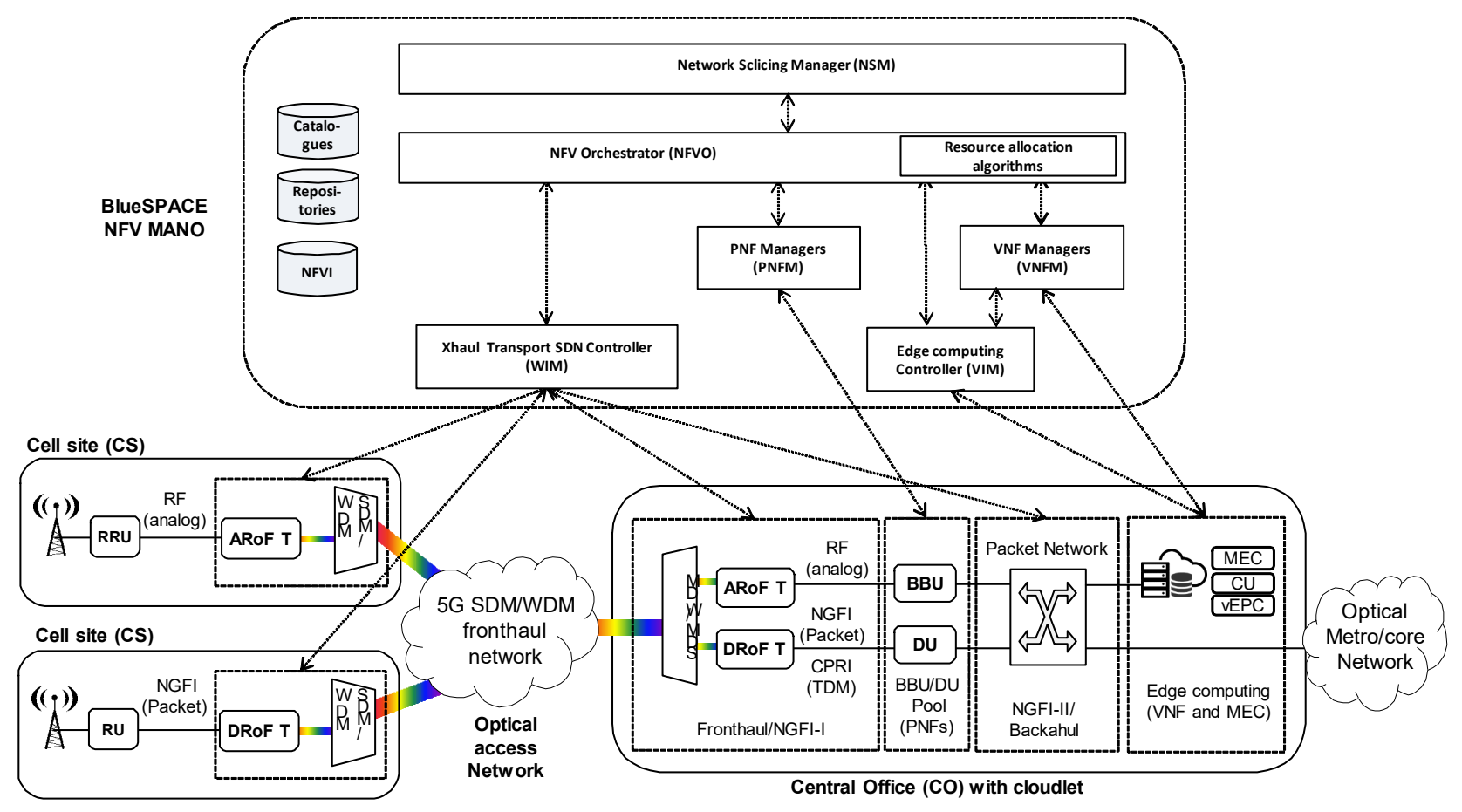

Figure 1: BlueSPACE's NFV MANO architectural framework

\section{OVERVIEW OF THE BLUESPACE'S NFV MANO ARCHITECTURAL FRAMEWORK}

The proposed NFV MANO system provides network services and network slicing services. In BlueSPACE, a network service is defined as the joint instantiation and configuration of VNFs and PNFs, and the required connections between different VNFs/PNFs to jointly realize a more complex function (e.g. service function chaining). On the other hand, a network slice is defined as a single network service instance or a concatenation of network service instances according to the 3GPP and ETSI SDOs [4].

The BlueSPACE's NFV MANO is deployed on top of the NFV infrastructure (NFVI) shown in Figure 1. It is composed of multiple cell sites connected to a central office (CO) with cloudlet through the 5G SDM/WDM-enabled fronthaul network with ARoF and DRoF transceivers. Additionally, the CO deploys a pool of BBUs/DUs (PNFs), a small DC (edge computing) acting as an NFV infrastructure point of presence (NFVI-PoP) deploying VNFs as well as MEC applications, and a packet network for backhaul/NGFI-II transport providing connectivity among the BBU/DUs, the small DC, and the optical metro/core network.

The ETSI NFV MANO identifies three functional blocks; virtualized infrastructure manager / WAN infrastructure manager (VIM/WIM), NFV orchestrator (NFVO) and VNF manager (VNFM). The VIM (typically implemented through the Openstack cloud controller) is responsible for controlling and managing the NFVI-PoP's virtualized compute, storage and networking resources, whilst the WIM (typically implemented through an SDN controller) is used to provision the transport connectivity. The VNFM is responsible for the lifecycle management of VNF instances (their instantiation, configuration, scaling up and down, termination), and the NFVO has two main responsibilities; the orchestration of NFV infrastructure resources across multiple VIMs and WIMs (resource orchestration), and the lifecycle management of network services (network service orchestration).

The BlueSPACE project proposes to extend the ETSI NFV MANO architecture with the following features required for the BlueSPACE project:

- SDN control of the 5G SDM/WDM-enabled fronthaul and packet backhaul networks: in BlueSPACE, in addition to the control of packet networks, it is also required to control the proposed SDM/WDM-enabled fronthaul network. The ETSI NFV MANO architecture considers that the WIM only controls packet transport networks. BlueSPACE will develop a holistic solution that integrates the control of optical spatial resources (SDM), optical spectral resources (WDM) and the statistical packet multiplexing in combination with $\mathrm{ARoF}$ and $\mathrm{DRoF}$ transceivers. The developed Transport SDN solution for the fronthaul and backhaul (known as xhaul) will be integrated with the NFVO, since currently the NFVO-WIM interface is not widely implemented and still lacking maturity [5].

- Integration of MEC with NFV: in BlueSPACE, MEC applications will be deployed in the edge computing of the CO. Despite there are some synergies between NFV and $\mathrm{MEC}$, since both require virtualized computing and storage resources to deploy the VNFs or the MEC applications, as well as connectivity services to connect the VNFs or MEC applications, currently MEC is not integrated in the NFV MANO framework. BlueSPACE will study the integration of MEC applications with NFV aiming at sharing the NFVI-PoPs and the NFV MANO stack. 


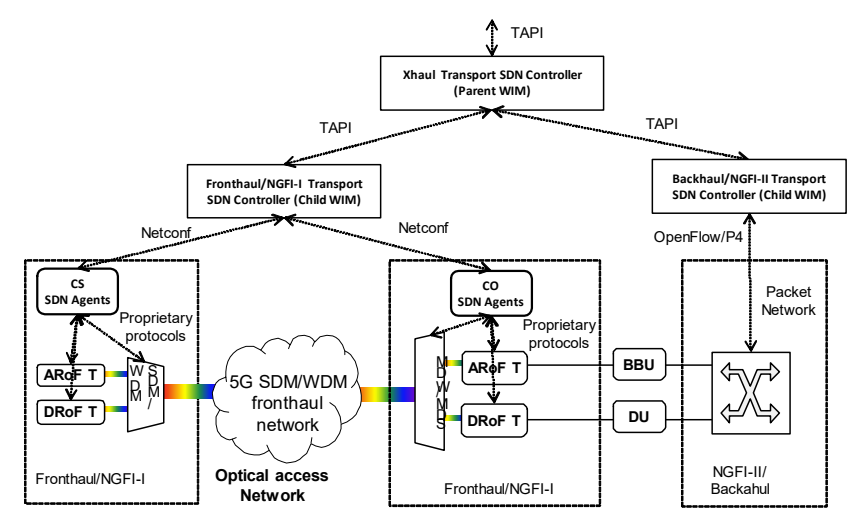

Figure 2: BlueSPACE's Xhaul Transport SDN control

- Management of virtual and physical network functions (VNFs and PNFS): In BlueSPACE, VNFs need to coexist with more traditional PNFs, while NFV network services need to be orchestrated considering jointly both type of network functions, i.e. PNFs and VNFs, each of them with their specific constraints, lifecycles, configuration and monitoring options, etc. The ETSI NFV MANO architecture considers that the PNF are externally managed by the Operations Support Systems (OSS). BlueSPACE will develop specific PNF managers (similar to VNF managers), as well as the agents that performs the actual configuration of the PNFs.

- Joint allocation of transport network and edge computing resources: In BlueSPACE, it is required to jointly perform the allocation of transport network resources (packet, WDM and SDM), as well as computing resources (i.e., virtual machines or containers) by the NFVO. Current NFV MANO framework considers the network as a commodity that provides packet pipes with QoS, either pre-provisioned or dynamically provisioned by the WIM between the end-points specified by the NFVO. Bluespace will extend NFVO and WIM to enable the computation and provisioning of strict network paths and cloud resource allocation algorithms.

- Multi-tenancy support (Network Slicing): In BlueSPACE, multi-tenancy and network slicing are adopted to improve the efficiency in the utilization of fronthaul resources and requires a deep integration between NFV MANO functions for service orchestration and SDN-based configuration of the transport networks. Current NFV MANO frameworks do not support network slicing. BlueSPACE will develop a Network Slice Manager (NSM), working on top of the NFVO and responsible for the lifecycle management of the network slices.

\section{XHAUL TRANSPORT SDN CONTROL}

A single SDN controller comprising the multiple and diverse technologies of the SDM/WDM-based fronthaul and packet-based backhaul is not realistic. Traditionally, network operators fragment their networks into multiple administrative domains for scalability, modularity, and security purposes. In BlueSPACE, we rely on a hierarchical Transport SDN control approach with different levels of hierarchy (parent/child architecture) as shown in Figure 2. In particular, we propose two SDN controllers (child WIMs), one for the fronthaul segment and another for the backhaul segment, and an SDN controller on top (parent WIM) acting as the Xhaul transport network orchestrator (or controller of controllers). It allows the control (e.g., E2E transport service provisioning, path computation, network topology and resource dissemination), at a higher, abstracted level, of the heterogeneous fronthaul and backhaul network technologies regardless of the specific control plane technology employed in each domain through the use of the Transport API (TAPI) defined in [6] and experimentally validated by the authors in [7]. This TAPI has to be properly extended in order to deal with the specific SDM transport technologies developed in BlueSPACE and that are currently not covered. It is used as the northbound Interface (NBI) of the child SDN controller and as SouthBound Interface (SBI) of a parent SDN controller. Additionally, the parent SDN controller also uses the TAPI as NBI with the NFVO. It is worth to highlight the considered architecture can be applied recursively enabling the cascading of the SDN controllers [8].

The child WIM for the backhaul network segment is based on a regular SDN controller for packets networks using OpenFlow or $\mathrm{P} 4$ protocols as SBI to configure the packet switches. On the fronthaul segment side, the proposed solution is to deploy SDN agents at the cell sites and another at the CO. The SDN agent's purpose is to map high-level operations coming from the child SDN controller into low-level, hardware-dependent operations using the proprietary protocols. This involves defining a data model for the ARoF and DRoF transceivers, and the optical SDM/WDM aggregation elements, and agreeing on a protocol, with the corresponding message formats and encodings. In BlueSPACE, we consider NETCONF protocol standardized by the IETF for network control and management. NETCONF relies on YANG as modelling language, used for defining the configurable parameters and state information in XML format. Thus, NETCONF and YANG provide a standard way to offer an open API for the child SDN controller's SBI. A first SDNenabled sliceable SDM-WDM transceiver controlled with YANG/NETCONF has been experimentally validated over a 11-km 6-mode 19-core fiber in [9].

In particular, we have implemented a YANG NETCONF server for the SDN agents of the nodes (i.e., cell sites and $\mathrm{CO}$ ), and a NETCONF client integrated as plugin in the child SDN controller. Specially, the YANG model describes a network node specifying NETCONF data model and protocol operations. The network node has an id, location and a list of components (e.g, ARoF, DRoF, WDM, SDM). Each component has its own parameters. The NETCONF client based on nnclient. Ncclient is a Python library for NETCONF clients. It aims to offer an intuitive API that sensibly maps the XML-encoded nature of NETCONF to Python constructs and idioms, and make writing network-management scripts easier. The NETCONF server used is Netopeer2. Netopeer2 is the 2nd generation of the well-known open-source NETCONF server that contains a modular datastore allowing interconnection and control of various applications via NETCONF protocol. In addition, it uses Sysrepo integrated with Netopeer2. Sysrepo is a YANG-based configuration and operational state data store. It provides a complete NETCONF management framework for Linux. 


\section{EDGE COMPUTING WITH INTEGRATED NFV AND MEC}

Existing NFV MANO frameworks allow to deploy network services composed of VNFs, dynamically instantiated in computing resources available in NFVI PoPs and selected according to resource-based constraints defined in the VNFD, e.g. vCPU, RAM and virtual disk requirements. Extending the concept of network services to include also MEC applications requires to enhance the NFVO functionalities in order to integrate the typical functions of a Mobile Edge Orchestrator (MEO) and operate over VIMs that handle not only generalized NFVI PoPs, but also MEC hosts.

The orchestration of MEC applications imposes additional constraints at the NFVO level. In particular, MEC applications may require the preliminary instantiation of specific MEC services to properly run in a given MEC host and, in turn, they may offer MEC services to be consumed by other applications. In a MEC architecture [10], the registration and discovery of available MEC services is typically handled through the Mobile Edge Platform that, in virtualized environments, becomes a VNF itself. Moreover, the instantiation of MEC applications is highly dependent on the geographical location of the target MEC host. For this reason, the traditional VNF placement algorithms at the NFVO must be enhanced to take into account this kind of constraints and the NFVI DB used as input for these algorithms should contain additional information about the characteristics of the available MEC hosts. As consequence, the advertisement of NFVI PoPs and available virtual resources performed on the OrVi interface between VIM and NFVO should now cover also the resource capabilities and the geographical position of MEC hosts and, jointly, their interconnectivity with the xhaul network and the PNFs deployed in the data plane.

Moreover, the NFVO should handle the dependencies between MEC applications and MEC services in an automated and seamless manner. In other terms, when deploying a MEC application that requires a set of MEC services, the NFVO should verify if these MEC services are already available in the target MEC host and, if not, it should implicitly instantiate MEC applications able to provide them. This requires a new level of interaction between NFVO and Mobile Edge Platform, possibly mediated through the Mobile Edge Platform Manager, in order to access the MEC service registry and coordinate the lifecycle management of the MEC applications.

\section{VNFS AND PNF MANAGERS}

The BlueSPACE NFV MANO framework should support the provisioning and management of different kinds of VNFs, integrating dedicated VNF Managers (VNFMs) that handles the VNF-specific interfaces for configuration and monitoring. However, beyond VNFs, BlueSPACE should also handle PNFs, for example to manage physical BBUs.

The introduction of PNFs in a network service brings additional challenges at the NFVO level, since the lifecycle of a PNF is much different than the typical lifecycle of a VNF. For example, a PNF cannot be dynamically instantiated or terminated or scaled up and down like a VNF, but it can be only started, stopped and configured usually through a proprietary, vendor-specific interface. Moreover, it cannot be attached to virtual networks with the same flexibility of a VNF, where virtual ports can be added dynamically to a VM and then interconnected to any virtual network. In fact, a PNF is deployed with a given number of network interfaces and they are interconnected to the physical network infrastructure in a static manner. Moreover, the PNF sharing capabilities may be quite restricted: their support usually depends on the specific characteristics of the PNF itself and this limits their re-usage in nested network services.

These specific characteristics impose several constraints on the management of single PNFs and on the orchestration of network services including PNFs, requiring the introduction of new components in the NFV MANO architecture, as well as the extensions in its interfaces, its information models and in the procedures of the already existing components. In particular:

- The NFVO should include a dedicated catalogue and record for PNFDs and PNF instances. The current PNFD, defined in [11], should be extended to describe additional constraints (e.g. sharing capacity), available actions, configurable parameters and capabilities of the PNF, as well as details about its network connectivity, not only in terms of logical connection points. The record of the PNF should include information about the current operational status (started, stopped) and configuration of the PNF, together with the references to the network service instances it belongs to, together with the related VNF Forwarding Graphs.

- $\quad$ The Or-Vi reference point [12] should be extended to advertise the PNFs deployed in the NFVI, with details about how their external connection points are interconnected to the physical network topology.

- $\quad$ The NFVO should be able to handle the lifecycle of VNFs and PNFs in different manners, consistently with the reduced set of actions that are allowed on the PNFs. At the NFVO Resource Orchestration function, resource allocation algorithms should be able to support PNFrelated constraints (e.g. about geographical position of the physical resources) and the configuration of the underlying transport network, where needed, must enable a suitable QoS in the connectivity towards the PNFs.

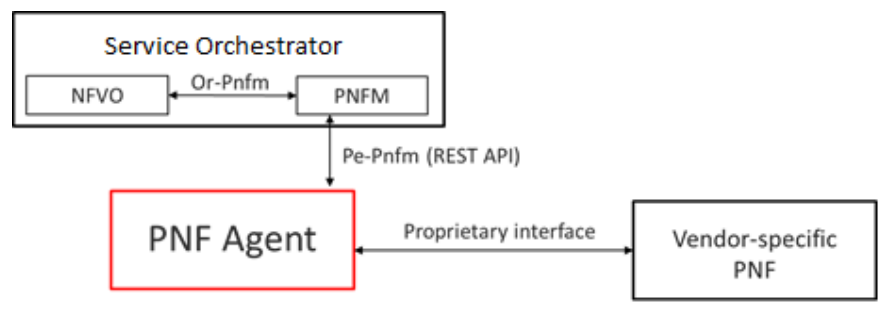

Figure 3: PNFM and PNF Agent in BlueSPACE's MANO architecture

- As shown in Figure 3, a new component, called PNF Manager (PNFM), is introduced in the architecture to provide functionalities similar to the VNFM's ones, but 
applicable to a PNF instead of a VNF. In other terms, the PNFM should manage the lifecycle of a PNF, taking into account the restricted set of actions that can be performed on the PNF itself. The PNFM should interact with the NFVO through a new reference point, called Or-Pnfm, which implements a subset of the methods specified for the Or-Vnfm reference point. For example, the Or-Pnfm reference point should support messages to start, stop and configure the PNF, as well as to collect monitoring data produced by the PNF. The implementation of the interface on the Or-Pnfm reference point can follow a standard protocol and data model, for example a REST API based on HTTP protocol and json messages, independent on the specific type of PNF.

- A new PNF-specific component, called PNF Agent, mediates between the PNFM and the PNF itself, implementing the client-side of the vendor-specific interface for the PNF management (e.g. via Telnet). On its NBI, the PNF Agent should interact with the PNFM over a new Pe-Pnfm reference point, which can support a subset of the methods specified for the $\mathrm{Ve}-\mathrm{Vnfm}$ reference point, including only the ones relevant for PNFs. The interface on the Pe-Pnfm may also be a REST API based on the HTTP protocol and json messages, still independent on the specific type of PNF.

\section{SERVice ORchestator (NFVO AND NSM)}

The BlueSPACE architecture adopts the concept of multitenancy and network slicing in order to deliver customized end-to-end services that are able to provide suitable levels of QoS while sharing the common physical infrastructure of the xhaul network.

Multi-tenancy is implemented through virtualization techniques and the reservation of dedicated and isolated resources to specific slices or services. The provisioning of QoS-enabled network connections at the underlying transport infrastructure is performed through the BlueSPACE SDN controller and it is fully integrated in the resource orchestration procedures at the NFVO.

The provisioning and management of network slices for customized services is handled through an additional component that operates on top of the NFVO and manages the association between network slices and NFV network services. In particular, a network slice can be considered as a logical entity, abstracted from the physical infrastructure, that consists of an orchestrated set of resources (network, computing, storage) and virtualized or physical network functions which are managed in a coordinated manner to delivered one or more services with some QoS characteristics, for example ultra-lowlatency or massive-connectivity machine communication services. Network slices can be dynamically created, scaled and terminated, based on the requirements and lifecycle of the services running within the slice.

In BlueSPACE, we refer to the 3GPP definition and modelling of network slices [13]: a Communication Service (CS) may be served by one or more Network Slice Instances (NSIs), where each NSI may be composed of more Network Slice Subnet Instances (NSSIs). NSIs and NSSIs are composed of Network Functions (NFs), which can be Physical Network Functions (PNFs) or Virtualized Network Functions (VNFs), i.e. NFs deployed on top of virtual resources. The lifecycle of network slices is not strictly bound to the lifecycle of the CSs; in particular, a NSI can exist independently on the existence of the CSs that are going to use it. In the architecture proposed by 3GPP, the provisioning and management of CSs and NSIs/NSSIs is handled through three functional components, implementing management functions for CSs, NSI and NSSI respectively. BlueSPACE defines the Network Slice Management Function (NSMF) component as a client of the NFVO, as suggested in [4]. The NSMF is in charge of managing the lifecycle of network slice instances and it translates their QoS requirements into suitable NFV network services which are instantiated and scaled on-demand at the NFVO, based on the evolution of the associated slice.

\section{CONCLUSIONS}

This paper has presented the BlueSPACE SDN/NFV architecture targeting a converged $\mathrm{x}$-haul (fronthaul/midhaul/backhaul) network and cloud infrastructure to offer network services and network slices for multi-tenancy.

\section{REFERENCES}

[1] Thomas Pfeiffer, Next Generation Mobile Fronthaul and Midhaul Architectures [Invited], Journal of Optical Communications and Netwrorks (JOCN), vol. 7, no. 11, pp. B38 - B45, November 2015.

[2] Chih-Lin I, Han Li, Jouni Korhonen, Jinri Huang, Jinri Huang, RAN Revolution with NGFI (xhaul) for 5G, Journal of Lightwave Technology, DOI: 10.1109/JLT.2017.2764924

[3] Network Functions Virtualisation (NFV); Management and Orchestration, ETSI GS NFV-MAN 001 v.1.1.1, (2014-12).

[4] Network Functions Virtualisation (NFV); Evolution and Ecosystem; Report on Network Slicing Support with ETSI NFV Architecture Framework, ETSI GR NFV-EVE 012 V0.0.9 (2017-07) - work in progress.

[5] Raul Muñoz, Ricard Vilalta, Ramon Casellas, Arturo Mayoral, Ricardo Martínez, Integrating Optical Transport Network Testbeds and Cloud Platforms to Enable End-to-End 5G and IoT Services, ICTON 2017.

[6] ONF technical recommendation, Functional Requirements for Transport API, ONF TR-527, June 2016.

[7] A. Mayoral, et al. "First experimental demonstration of distributed cloud and heterogeneous network orchestration with a common Transport API for E2E services with QoS." Optical Fiber Conference (OFC) 2016.

[8] A. Mayoral, R. Vilalta, R. Muñoz, R. Casellas, R. Martínez, V. López, Cascading of tenant SDN and cloud controllers for 5G network slicing using Transport API and Openstack API, in Proceedings of International Conference on Optical Fiber Communications (OFC), 19-23 March 2017, Los Angeles (USA).

[9] SDN-enabled Sliceable Multi-dimensional (Spectral and Spatial) Transceiver Controlled with YANG/NETCONF, in Proceedings of International Conference on Optical Fiber Communications (OFC), 1115 March, San Diego (USA).

[10] ETSI GS MEC 003, "Mobile Edge Computing (MEC); Framework and Reference Architecture", v1.1.1, March 2016

[11] ETSI GS NFV-IFA014 (V2.3.1): "Network Functions Virtualisation (NFV) Release 2; Management and Orchestration; Network Service Templates Specification", August 2017

[12] ETSI GS NFV-IFA005 (V2.3.1): "Network Functions Virtualisation (NFV) Release 2; Management and Orchestration; Or-Vi reference point - Interface and Information Model Specification", August 2017

[13] 3GPP, "Study on management and orchestration of network slicing for next generation network (Release 15)", 3GPP TR 28.801 2.0.1, September 2017 